Letter

\section{Employment and Functional Ability in Men and Women With Axial Spondyloarthritis as Described in the US-based Corrona Psoriatic Arthritis/Spondyloarthritis Registry}

\section{To the Editor:}

We read with interest the recent article from Mease et $\mathrm{al}^{1}$ regarding the analysis of men and women with axial spondyloarthritis (axSpA) captured in the Corrona Psoriatic Arthritis/ Spondyloarthritis Registry. Dedicated comparative analysis in large, well-characterized cohorts such as this are crucial to identifying the variation of axSpA between males and females.

The data on employment status including outcomes from the Work Productivity and Activity Impairment Questionnaire (WPAI) were especially interesting. It was reported that the rate of employment was similar between men and women $(67.8 \%$ vs $64 \%, P=0.39)$. Females had a significantly lower rate of disability (12.6\% vs $16 \%, P<0.01$ ), despite reporting worse impairment while working $(35.4 \%$ vs $24.9 \%, P<0.01)$, greater impairment of activity $(45.9 \%$ vs $36.1 \%, P=0.03)$, and worse overall impairment $(28.6 \%$ vs $27.1 \%, P<0.01)$. Of the WPAI domains analyzed, the only domain that was similar between sexes was the percentage of work time missed $(7.3 \%$ vs $6.7 \%, P=0.33)$. These findings likely contribute to the observed higher frequency of women working part-time $(10.5 \%$ vs $3.6 \%, P<0.01)$ and the lower frequency of women with axSpA working full time compared to men $(53.7 \%$ vs $62.1 \%, P<0.01)$.

These findings indicated a high prevalence of presenteeism despite worse level of functional impairment observed in women with axSpA. Loss of work productivity can result in work instability, ${ }^{2}$ which can be a source of significant physical and mental distress. As such, the significantly higher observed prevalence of depression in women in this study $(25.7 \%$ vs $12.1 \%, P<0.01)$ is another interesting factor to consider. In the general population, work instability has been shown to contribute to psychological distress, ${ }^{3}$ whereas conversely, work productivity has been shown to be lower in patients with known depression. ${ }^{4}$ In axSpA, work productivity loss remains a challenge in management, with high rates of presenteeism observed. ${ }^{5}$

This raises a number of additional questions. Is the higher prevalence of depression in women with axSpA driving the worse level of function and decreased work productivity? Or could the worse limitation in functional ability observed in women with axSpA lead to work instability and depression? Are women with axSpA under greater pressure to remain in the workforce or less able to qualify for disability?

Further research to examine workforce participation and the effect of mental health is needed in axSpA, especially in terms of how this varies between men and women. Identification of the issue that triggers this series of negative consequences, eventu- ally resulting in depression and unemployment, is the first step in improving detection of at-risk patients with axSpA. Prompt detection would allow intervention and provision of supportive services that can prevent progression to loss of work and longterm mental health issues. ${ }^{6}$ Further, improved understanding of sex-specific relationships between mental health and work productivity in axSpA would encourage greater resource allocation to supportive services. Detailed, high-quality data collection with comparative analysis between sexes in axSpA, such as that seen in the Corrona Psoriatic Arthritis/Spondyloarthritis Registry, offers significant insight into the real-world effects of these issues in axSpA. However, development of longitudinal data is needed to determine presence of a causal relationship between functional ability, work productivity, and mental health in axSpA to truly understand the effects on both men and women with the disease.

Sinead Maguire ${ }^{1,2}$ (D), MB BCh, BAO, MRCPI

Gillian Fitzgerald ${ }^{3}$ (D) $\mathrm{MB}$ BCh, BAO, MRCPI, PhD

Finbar O'Shea ${ }^{1,2}$ (D), MB BCh, BAO, MRCPI

${ }^{1}$ Department of Rheumatology, St James' Hospital, Dublin;

${ }^{2}$ School of Medicine, Trinity College Dublin, Dublin;

${ }^{3}$ Department of Rheumatology, Galway University Hospitals, Galway, Ireland.

$\mathrm{SM}$ is the recipient of the Gilead Inflammation Fellowship.

GF has received support for educational events from $\mathrm{UCB}$ and has been on an advisory board for AbbVie. SM and FO declare no conflicts of interest relevant to this article.

Address correspondence to Dr. S. Maguire, Department of Rheumatology, St James' Hospital, Dublin, Ireland. Email: sineadmaguire@rcsi.ie.

\section{REFERENCES}

1. Mease PJ, McLean RR, Dube B, Liu M, Rebello S, Glynn M, et al. Comparison of men and women with axial spondyloarthritis in the US-based Corrona Psoriatic Arthritis/Spondyloarthritis Registry. J Rheumatol 2021;48:1528-36.

2. Tillett W, Shaddick G, Askari A, Cooper A, Creamer P, Clunie $G$, et al. Factors influencing work disability in psoriatic arthritis: first results from a large UK multicentre study. Rheumatology 2015;54:157-62

3. Schneider D, Harknett K. Consequences of routine work-schedule instability for worker health and well-being. Am Sociol Rev 2019;84:82-114.

4. Woo JM, Kim W, Hwang TY, Frick KD, Choi BH, Seo YJ, et al. Impact of depression on work productivity and its improvement after outpatient treatment with antidepressants. Value Health 2011;14:475-82

5. Nikiphorou E, Ramiro S. Work disability in axial spondyloarthritis. Curr Rheumatol Rep 2020;22:55.

6. Grimani A, Aboagye E, Kwak L. The effectiveness of workplace nutrition and physical activity interventions in improving productivity, work performance and workability: a systematic review. BMC Public Health 2019;19:1676. 